\title{
Diagnóstico de enfermagem síndrome de terminalidade em idosos com demência avançada: mapeamento cruzado
}

\author{
Nursing diagnosis of terminal syndrome in elderly with advanced dementia: cross mapping \\ Diagnóstico de enfermería del síndrome terminal en ancianos con demencia avanzada: mapeo cruzado
}

\author{
Dayana Medeiros do Amaral Passarelles' @; Rosimere Ferreira Santana' $@$; Antonia Rios Almeida' $@$;

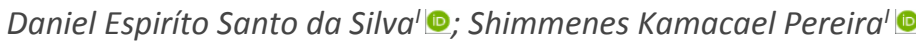

'Universidade Federal Fluminense, Niterói, RJ, Brasil

\begin{abstract}
RESUMO
Objetivo: identificar elementos da Síndrome de terminalidade a partir do cruzamento de termos registrados pelos enfermeiros no cuidado ao fim de vida em idosos com demência avançada. Método: estudo observacional, retrospectivo, da ferramenta metodológica mapeamento cruzado. Foram coletados registros dos últimos 10 dias de vida de 38 prontuários de pessoas com demência avançada. Resultados: foram identificados 97 termos de respostas humanas, e através do mapeamento cruzado, foram elencados 22 diagnósticos de enfermagem, desses 11 diagnósticos de enfermagem apresentaram relevância estatística em $50 \%$ ou mais dos pacientes e oito diagnósticos de enfermagem apresentaram-se relevantes estatisticamente quando avaliados de acordo com a prevalência nas 380 observações. A Síndrome de terminalidade foi verificada em todas 380 avaliações, em média 7,5 diagnósticos da síndrome foram observados. Conclusão: a alta prevalência da Síndrome de terminalidade sustenta a inclusão do diagnóstico de enfermagem na Taxonomia II da NANDA-I, dado que os enfermeiros já a observam e a registram em sua prática.

Descritores: Diagnóstico de Enfermagem; Cuidados Paliativos; Cuidados Paliativos na Terminalidade da Vida; Enfermagem Geriátrica.
\end{abstract}

\section{ABSTRACT}

Objective: to identify elements of Terminal Syndrome by cross-referencing terms recorded by nurses providing end-of-life care for elderly people with advanced dementia. Method: in this retrospective, observational study, using a cross-mapping methodological tool, records of the last 10 days of life were collected from 38 medical records of people with advanced dementia. Results: 97 human-response terms were identified, and by cross-mapping, 22 nursing diagnoses were listed; of these, 11 nursing diagnoses displayed statistical importance in $50 \%$ or more of the patients, while eight nursing diagnoses were statistically important when assessed by prevalence in the 380 observations. Terminal Syndrome was found in all 380 evaluations, averaging 7.5 diagnoses of the syndrome. Cases were observed Conclusion: the high prevalence of Terminal Syndrome supports the inclusion of this nursing diagnosis in the NANDA-I Taxonomy II, as nurses already observe and record in practice.

Descriptors: Nursing Diagnosis; Palliative Care; Hospice Care; Geriatric Nursing.

\section{RESUMEN}

Objetivo: identificar elementos del síndrome terminal mediante términos de referencia cruzada registrados por enfermeras que brindan atención al final de la vida a personas mayores con demencia avanzada. Método: en este estudio observacional retrospectivo, utilizando una herramienta metodológica de mapeo cruzado, se recolectaron registros de los últimos 10 días de vida de 38 historias clínicas de personas con demencia avanzada. Resultados: Se identificaron 97 términos de respuesta humana y, mediante mapeo cruzado, se enumeraron 22 diagnósticos de enfermería; de estos, 11 diagnósticos de enfermería mostraron importancia estadística en el $50 \%$ o más de los pacientes, mientras que ocho diagnósticos de enfermería fueron estadísticamente importantes cuando se evaluaron por prevalencia en las 380 observaciones. El síndrome terminal se encontró en las 380 evaluaciones, con un promedio de 7,5 diagnósticos del síndrome. Se observaron casos Conclusión: la alta prevalencia de Síndrome Terminal apoya la inclusión de este diagnóstico de enfermería en la Taxonomía II de NANDA-I, ya que las enfermeras ya observan y registran en la práctica.

Descriptores: Diagnóstico de Enfermería; Cuidados Paliativos; Cuidados Paliativos al Final de la Vida; Enfermería Geriátrica.

\section{INTRODUÇÃO}

Segundo a Organização Mundial de Saúde, os cuidados paliativos definem-se como uma abordagem que promove a qualidade de vida de pacientes e seus familiares, que enfrentam doenças que ameacem a continuidade da vida, através da prevenção e alívio do sofrimento através da identificação precoce, avaliação e tratamento da dor e outros problemas de natureza física, psicossocial e espiritual ${ }^{1}$.

A necessidade dos cuidados paliativos para pacientes com câncer foi reconhecida mundialmente, porém atualmente discute-se sua necessidade para doenças crônicas não oncológicas ${ }^{2}$. Logo, observa-se que as publicações referentes aos cuidados paliativos e cuidados ao fim da vida direcionam-se em sua maioria para pacientes com câncer, porém há um aumento progressivo de estudos nos últimos anos referentes à população de idosos com demência avançada, devido às estimativas globais de crescimento da população idosa ${ }^{3,4}$. 
A demência possui um curso diferente comparado ao câncer, pois pacientes com câncer podem experimentar um declínio substancial na funcionalidade nas últimas semanas de vida e prognóstico bem definido. Enquanto pacientes com demência possuem um declínio cognitivo, funcional e físico mais prolongado com difícil prognóstico ${ }^{3,5}$. Contanto, a quantidade de sintomas no cuidado ao fim de vida no paciente com demência pode ser equivalente à quantidade de sintomas vivenciados pelo paciente com câncer, porém o tempo da prevalência desses sintomas será prolongado devido às características da própria doença ${ }^{6}$.

Os cuidados ao fim de vida devem ser realizados por uma equipe interdisciplinar, e nela o enfermeiro deve possuir habilidades voltadas para a avaliação sistemática dos sinais e sintomas e promoção de conforto 3 . Porém, durante o controle de sintomas nos cuidados ao fim de vida em idosos com demência, os enfermeiros possuem dificuldade de elucidar e caracterizar os diagnósticos de enfermagem, devido à multiplicidade de diagnósticos de enfermagem que podem surgir.

Para tanto, elaborou-se uma proposta diagnostica de enfermagem, sindrômica denominada Síndrome de terminalidade definida como: Estado de deterioração de um conjunto de sinais e sintomas físicos, psicológico, social e espiritual do indivíduo decorrente do agravo da doença. Foram atribuídas como características definidoras: Dor crônica, Distúrbio no padrão de sono, Náusea, Nutrição desiquilibrada: menor que as necessidades corporais, Ansiedade, Fadiga, Padrão respiratório ineficaz, Conforto prejudicado, Sofrimento espiritual, Termorregulação ineficaz, Constipação, Diarreia, Confusão crônica, Retenção urinária, Pesar, Mobilidade física prejudicada, Integridade da pele prejudicada, Integridade tissular prejudicada, Volume de líquidos excessivo. Fatores relacionados foram: Cuidados paliativos, Doenças crônicas em estágio avançado e Incapacidade física crônica. População de risco: idosos. E Condições associadas: Cuidados ao fim de vida.

Todavia, delimitou-se como objetivo: identificar elementos da Síndrome de terminalidade a partir do cruzamento de termos registrados pelos enfermeiros nos cuidados ao fim de vida em idosos com demência avançada.

\section{MÉTOdo}

Trata-se de um estudo observacional retrospectivo, de mapeamento cruzado, o qual possibilitou mapear e comparar os registros de enfermagem não padronizados com terminologias uniformizadas, seguindo-se as regras propostas por Lucena e Barros ${ }^{7}$.

O estudo foi realizado em um hospital especializado em cuidados paliativos, fundamentado no modelo de cuidados transicionais. É um hospital de cuidados extensivos que oferece acompanhamento integral aos pacientes de reabilitação, cuidados continuados, e cuidados paliativos.

Os critérios de inclusão foram: idade maior ou igual a 18 anos; em abordagem nos cuidados ao fim de vida; com ou sem tratamento curativo prévio; tempo mínimo de 10 dias de internação com o desfecho de óbito; com data de internação de 2013 a dezembro de 2017; ambos os períodos foram escolhidos devido ao ano de inauguração de cada unidade, 2013 para a unidade de Niterói, e de 2016 para unidade de Botafogo. O tempo de 10 dias de internação foi escolhido, pois não foi encontrado na literatura um consenso na quantidade de dias que se pode considerar como fim de vida para pacientes com demência avançada.

De acordo com a delimitação temporal, encontraram-se disponíveis 55 prontuários de indivíduos em cuidados ao fim de vida, com desfecho de óbito, porém 17 prontuários foram excluídos por possuírem tempo inferior a 10 dias de internação, portanto, a amostra final foi composta por 38 prontuários. O período de coleta de dados foi de setembro à dezembro de 2018.

O tempo de 10 dias de acompanhamento foi escolhido para possibilitar uma análise da prevalência das características definidoras da "Síndrome de terminalidade" ao longo dos últimos dias de vida. Além disso, a literatura não possui um consenso da quantidade exata para considerar como definição para cuidados ao fim de vida.

O instrumento de coleta de dados compunha: dados de identificação do paciente e histórico prévio; coleta dos registros dos enfermeiros; termos livres identificados. Após o preenchimento de cada instrumento e mapeamento dos termos livres, foi realizado a inferência dos diagnósticos de enfermagem por três avaliadores, incluindo o pesquisador, um perito em diagnóstico de enfermagem, e outro em cuidados paliativos de modo independentes, isso, para evitar um viés de seleção dos diagnósticos de enfermagem. E por fim, realizou-se, encontro presencial para consenso dos diagnósticos elencados.

$\mathrm{Na}$ etapa inicial de coleta de dados foram identificados 176 termos livres, esses foram organizados, excluindo-se os termos que denotavam resultados e intervenções de enfermagem, a amostra final foi de 97 termos livres de enfermagem.

Na segunda etapa foram avaliados termos de enfermagem e seu cruzamento com os diagnósticos de enfermagem individualmente pelos três peritos. Ao todo foram elencados 44 diagnósticos de enfermagem, porém após reunião de 
consenso e discussões sobre raciocínio crítico e diagnóstico, seleção de prioridades frente a abordagem em cuidados ao fim de vida, obteve-se 22 diagnósticos de enfermagem.

Para realizar a discussão e inferência dos diagnósticos de enfermagem pelos peritos, considerou-se a comparação dos termos destacados com a definição dos diagnósticos, características definidoras, fatores relacionados, condições associadas e populações em risco.

Após o consenso entre os peritos foi criado um banco de dados em planilha eletrônica com os dados de caracterização dos indivíduos, os termos mapeados e os diagnósticos de enfermagem. Os termos cruzados com diagnósticos de enfermagem foram tabulados e organizados de acordo com a análise da prevalência dos 10 dias, com variáveis de caracterização tabuladas por pacientes. Os dados foram analisados pelo programa SPSS (Statistical Package for the Social Science), versão 22.0

O estudo foi aprovado pelo Comitê de Ética em Pesquisa da instituição, de acordo com parecer no 2.799.814.

\section{RESULTADOS}

A maioria da população era do sexo feminino 28 (73,7\%). A média de idade é de 84,7 anos, com faixa etária predominante de 80 a 102 anos (81,6\%). Os pacientes dessa amostra eram predominantemente brasileiros (78,9\%), e possuíam demência em estágio avançado $36(94,7 \%)$, ou doenças neurológicas crônicas como esclerose múltipla e astrocitoma. A maioria teve como procedência o hospital 31 (81,6\%), ou seja, tratam de pacientes que estavam com internações de longo prazo no hospital.

Foram identificados 97 termos referentes as respostas humanas. A tabela 1 apresenta a prevalência dos termos livres com frequência significativa (>50\%).

TABELA 1: Prevalência dos termos de registros de enfermeiros nos pacientes em cuidados ao fim de vida. Niterói, Rio de Janeiro, Brasil, 2019.

\begin{tabular}{|c|c|c|c|c|c|c|c|c|c|c|c|c|}
\hline Termos & 1 & 2 & 3 & 4 & 5 & 6 & 7 & 8 & 9 & 10 & $\begin{array}{c}\text { Total de } \\
\text { observações } \\
\% \\
\end{array}$ & $\begin{array}{c}\text { Total por } \\
\text { paciente } \\
\% \\
\end{array}$ \\
\hline Pouca interação & 68,4 & 65,8 & 73,7 & 65,8 & 78,9 & 73,7 & 81,6 & 78,9 & 84,2 & 86,8 & 75,8 & 89,5 \\
\hline Sonolência & 42,1 & 44,7 & 47,4 & 50,0 & 47,4 & 44,7 & 50,0 & 55,3 & 52,6 & 44,7 & 47,8 & 84,2 \\
\hline Dieta zero & 36,8 & 28,9 & 34,2 & 36,8 & 36,8 & 39,4 & 44,7 & 65,8 & 73,7 & 65,8 & 46,3 & 81,6 \\
\hline Constipação & 39,5 & 36,8 & 36,8 & 39,5 & 44,7 & 52,6 & 47,4 & 47,4 & 52,6 & 55,3 & 45,3 & 78,9 \\
\hline Acamada & 60,5 & 60,5 & 63,2 & 60,2 & 63,2 & 60,5 & 63,2 & 60,5 & 60,5 & 63,2 & 61,6 & 68,4 \\
\hline Fragilidade capilar & 47,4 & 52,6 & 50,0 & 52,6 & 52,6 & 52,6 & 50,0 & 55,3 & 52,6 & 47,4 & 51,3 & 65,8 \\
\hline Edema & 47,5 & 50,0 & 42,1 & 44,7 & 42,1 & 50,0 & 47,4 & 42,1 & 42,1 & 47,4 & 45,5 & 63,2 \\
\hline $\begin{array}{l}\text { Desconforto } \\
\text { respiratório }\end{array}$ & 2,6 & 0 & 13,2 & 7,9 & 7,9 & 13,2 & 18,2 & 21,1 & 28,9 & 39,5 & 15,3 & 60,5 \\
\hline Secretivo & 36,8 & 31,6 & 42,1 & 42,1 & 44,7 & 39,5 & 42,1 & 47,4 & 47,4 & 36,8 & 41,1 & 60,5 \\
\hline $\begin{array}{l}\text { Eliminação urinária } \\
\text { ausente }\end{array}$ & 5,3 & 5,3 & 5,3 & 15,8 & 7,9 & 15,8 & 23,7 & 23,7 & 28,9 & 36,8 & 16,8 & 57,9 \\
\hline $\begin{array}{l}\text { Baixo débito } \\
\text { urinário }\end{array}$ & 21,1 & 26,3 & 26,3 & 31,6 & 28,9 & 31,6 & 21,1 & 26,3 & 28,9 & 18,4 & 26,1 & 55,3 \\
\hline Torporoso & 10,5 & 21,1 & 15,8 & 21,1 & 28,9 & 21,1 & 36,8 & 26,3 & 34,2 & 31,6 & 24,7 & 55,3 \\
\hline Resíduo gástrico & 26,3 & 28,9 & 21,1 & 23,7 & 21,1 & 21,1 & 26,3 & 26,3 & 26,3 & 15,8 & 23,7 & 55,3 \\
\hline Hipocorado & 34,2 & 31,6 & 28,9 & 31,6 & 31,6 & 34,2 & 31,6 & 36,8 & 39,5 & 39,5 & 33,9 & 52,6 \\
\hline Sedado & 10,5 & 10,5 & 13,2 & 15,8 & 15,8 & 18,4 & 23,7 & 42,1 & 47,4 & 50,0 & 24,8 & 52,6 \\
\hline Dor & 34,2 & 23,7 & 23,7 & 18,4 & 23,7 & 28,9 & 13,2 & 21,1 & 23,7 & 13,2 & 22,4 & 50 \\
\hline Lesão por pressão & 47,4 & 44,7 & 44,7 & 44,7 & 42,1 & 42,1 & 44,7 & 44,7 & 44,7 & 42,1 & 44,2 & 50 \\
\hline Hiperemia & 36,8 & 36,8 & 34,2 & 39,5 & 36,8 & 36,8 & 39,5 & 36,8 & 28,9 & 28,9 & 35,5 & 50 \\
\hline Hipotensão & 18,4 & 7,9 & 13,2 & 10,5 & 21,1 & 15,8 & 23,7 & 18,4 & 26,3 & 36,8 & 19,2 & 50 \\
\hline
\end{tabular}

De acordo com a análise, 19 termos foram considerados com prevalência de 50\% em alguma das 10 observações, e três foram considerados prevalentes em todos os 10 dias observados: Pouca interação, Acamada e Fragilidade capilar.

Os termos Desconforto respiratório, Secretivo, Eliminação urinária ausente, Baixo débito urinário, Torporoso, Resíduo gástrico, Hipocorado, Dor, Lesão por pressão, Hiperemia e Hipotensão não apresentaram significância estatística ao longo dos dias e no total de observações. Porém observa-se que esses termos estiveram presentes nos últimos dias de vida, o que pode sugerir que apesar de presentes obtiveram resolução, que não foram prevalentes ao longo dos dias, e que podem ser mais específicos para os últimos momentos de vida. 
A tabela 2 exibe o cruzamento desses termos com os diagnósticos de enfermagem propostos pela Taxonomia II da NANDA-I.

TABELA 2: Prevalência dos diagnósticos de enfermagem no total de pacientes, total de observações e em cada uma das 10 observações, Niteró, Rio de Janeiro, Brasil, 2019.

\begin{tabular}{|c|c|c|c|c|c|c|c|c|c|c|c|c|}
\hline $\begin{array}{l}\text { Diagnóstico de } \\
\text { enfermagem }\end{array}$ & 1 & 2 & 3 & 4 & 5 & 6 & 7 & 8 & 9 & 10 & $\begin{array}{c}\text { Total de } \\
\text { observa- } \\
\text { ções \% }\end{array}$ & $\begin{array}{c}\text { Total por } \\
\text { pacien- } \\
\text { te } \% \\
\end{array}$ \\
\hline $\begin{array}{l}\text { Nutrição } \\
\text { desequilibrada: menor } \\
\text { do que as necessidades } \\
\text { corporais }\end{array}$ & 78,9 & 68,4 & 81,5 & 73,7 & 71,1 & 76,3 & 76,3 & 86,8 & 86,8 & 81,5 & 78,2 & 97,4 \\
\hline $\begin{array}{l}\text { Distúrbio no padrão de } \\
\text { sono }\end{array}$ & 57,9 & 65,8 & 63,2 & 68,4 & 60,5 & 68,4 & 73,7 & 73,7 & 81,5 & 84,2 & 69,7 & 94,7 \\
\hline $\begin{array}{l}\text { Mobilidade física } \\
\text { prejudicada }\end{array}$ & 92,1 & 92,1 & 94,7 & 92,1 & 94,7 & 92,1 & 92,1 & 92,1 & 89,5 & 84,2 & 91,6 & 94,7 \\
\hline $\begin{array}{l}\text { Padrão respiratório } \\
\text { ineficaz }\end{array}$ & 47,4 & 36,8 & 52,6 & 57,9 & 60,5 & 57,9 & 65,8 & 65,8 & 73,7 & 78,9 & 59,7 & 94,7 \\
\hline $\begin{array}{l}\text { Integridade da pele } \\
\text { prejudicada }\end{array}$ & 71,1 & 76,3 & 71,1 & 76,3 & 78,9 & 76,3 & 81,5 & 78,9 & 73,7 & 68,4 & 75,3 & 89,5 \\
\hline $\begin{array}{l}\text { Integridade tissular } \\
\text { prejudicada }\end{array}$ & 84,2 & 81,5 & 81,5 & 78,9 & 81,5 & 76,3 & 78,9 & 73,7 & 78,9 & 78,9 & 79,5 & 86,8 \\
\hline Constipação & 44,7 & 52,6 & 47,4 & 50 & 55,3 & 57,9 & 60,5 & 63,2 & 65,8 & 63,2 & 56,1 & 78,9 \\
\hline $\begin{array}{l}\text { Perfusão tissular } \\
\text { periférica ineficaz }\end{array}$ & 42,1 & 34,2 & 31,6 & 39,5 & 39,5 & 44,7 & 44,7 & 47,8 & 55,3 & 57,9 & 43,7 & 76,3 \\
\hline Retenção urinária & 26,3 & 31,5 & 31,5 & 39,5 & 34,2 & 44,7 & 39,5 & 47,8 & 55,3 & 50 & 40 & 76,3 \\
\hline Confusão crônica & 57,9 & 57,9 & 63,2 & 57,9 & 63,2 & 60,5 & 60,5 & 65,8 & 60,5 & 63,2 & 61,1 & 71,1 \\
\hline $\begin{array}{l}\text { Volume de líquidos } \\
\text { excessivo }\end{array}$ & 50 & 50 & 42,1 & 44,7 & 42,1 & 50 & 47,8 & 42,1 & 42,1 & 47,8 & 45,8 & 63,2 \\
\hline Dor crônica & 31,6 & 21,1 & 23,7 & 18,4 & 23,7 & 28,9 & 13,2 & 18,4 & 23,7 & 13,2 & 21,6 & 44,7 \\
\hline Pesar & 13,2 & 13,2 & 18,4 & 21,1 & 10,5 & 15,8 & 10,5 & 21,1 & 21,1 & 18,4 & 16,3 & 42,1 \\
\hline Náusea & 10,5 & 13,2 & 2,6 & 10,5 & 13,2 & 7,9 & 5,3 & 7,9 & 2,6 & 5,3 & 7,9 & 31,6 \\
\hline Fadiga & 7,9 & 10,5 & 7,9 & 7,9 & 13,2 & 5,3 & 13,2 & 7,9 & 13,2 & 13,2 & 10 & 28,9 \\
\hline $\begin{array}{l}\text { Termorregulação } \\
\text { ineficaz }\end{array}$ & 10,5 & 0 & 2,6 & 0 & 2,6 & 0 & 10,5 & 5,3 & 7,9 & 7,9 & 4,7 & 28,9 \\
\hline Ansiedade & 5,3 & 5,3 & 5,3 & 10,5 & 10,5 & 7,9 & 0 & 2,6 & 5,3 & 2,6 & 5,5 & 23,7 \\
\hline $\begin{array}{l}\text { Volume de líquidos } \\
\text { deficiente }\end{array}$ & 18,4 & 21,1 & 21,1 & 18,4 & 18,4 & 21,1 & 18,4 & 18,4 & 16,8 & 10,5 & 18,2 & 23,7 \\
\hline Conforto prejudicado & 2,6 & 2,6 & 5,3 & 2,6 & 13,2 & 10,5 & 0 & 5,3 & 5,3 & 2,6 & 5 & 21,1 \\
\hline $\begin{array}{l}\text { Integridade da } \\
\text { membrana mucosa oral } \\
\text { prejudicada }\end{array}$ & 7,1 & 13,2 & 13,2 & 13,2 & 13,2 & 13,2 & 13,2 & 13,2 & 13,2 & 10,5 & 12,4 & 18,4 \\
\hline Diarreia & 7,9 & 5,3 & 5,3 & 7,9 & 5,3 & 7,9 & 2,6 & 7,9 & 2,6 & 5,3 & 5,8 & 15,8 \\
\hline Dor aguda & 2,6 & 2,6 & 0 & 0 & 0 & 0 & 0 & 2,8 & 0 & 0 & 0,8 & 5,3 \\
\hline
\end{tabular}

Observa-se que oito diagnósticos de enfermagem se apresentaram como relevantes: Nutrição desequilibrada: menor do que as necessidades corporais, Distúrbio no padrão de sono, Mobilidade física prejudicada, Padrão respiratório ineficaz, Integridade da pele prejudicada, Integridade tissular prejudicada, Constipação e Confusão crônica. O termo Dor foi identificado em $50 \%$ da amostra, porém ao considerar o tempo destaca-se Dor Crônica $(44,7 \%)$.

Dos diagnósticos de enfermagem mapeados para os pacientes em cuidados ao fim de vida, somente os diagnósticos Perfusão tissular periférica ineficaz, Dor aguda, Integridade da membrana mucosa oral prejudicada e Volume de líquidos deficiente não fazem parte do grupo de características definidoras da Síndrome de terminalidade. Da Síndrome de terminalidade, somente o diagnóstico Sofrimento Espiritual não foi registrado.

Em todos os dias e total de observações, a quantidade de diagnósticos da síndrome apresentava baixa variabilidade $(0,20=<\mathrm{CV}=<0,40)$. No total, independente da avaliação, a quantidade de diagnósticos por paciente variou de 3 a 13 diagnósticos, com média de 7,5 diagnósticos por paciente, desvio padrão de 1,9, que resultou num $\mathrm{CV}=0,25$ (baixa variabilidade). Em cada avaliação as estatísticas não se diferem muito das estatísticas globais e não há diferença significativa entre as distribuições de quantidades de diagnósticos da Síndrome das distintas avaliações ( $p$ - 
valor=0,597 do teste de Friedman). Logo, a Síndrome de terminalidade foi verificada em $100 \%$ das 380 avaliações com média de 7,5 diagnósticos por pacientes, em seus 10 últimos dias de vida.

\section{DISCUSSÃO}

O principal dado desse estudo é a demonstração da presença da Síndrome de terminalidade, a partir dos termos descritos no prontuário do paciente, por enfermeiros da prática clínica, em cuidados paliativos não oncológicos aos idosos com demência avançada. A alta prevalência de uma complexidade de diagnósticos de enfermagem ocorridos simultaneamente no cuidado ao fim de vida demonstra a relevância desse diagnóstico na prática de enfermagem.

Além da prevalência de idosos com demência avançada, obteve-se também uma média de idade alta com 84,7 anos. E isso influencia a carga global de sofrimento grave relacionado com a saúde, pois uma variedade de alterações fisiológicas que ocorrem nas últimas horas e dias de vida ${ }^{8}$. E, essas geralmente são acompanhadas por declínio funcional e por uma multiplicidade de sintomas, como os encontrados nesse estudo através do mapeamento dos termos livres ${ }^{9-12}$.

Prognosticar e definir a fase de fim de vida na demência avançada é um desafio para a equipe de saúde, e mais difícil que no câncer, devido às características da própria doença de baixa funcionalidade, comprometimento cognitivo grave, múltiplas comorbidades, fragilidade avançada e falência orgânica ${ }^{3,5,13-15}$. Os pacientes com demência avançada e outras doenças neurológicas crônicas apresentam os sintomas ao fim de vida por um período mais prolongado do que em pacientes com câncer. Visto que nesse estudo, a prevalência da Síndrome de terminalidade esteve nos 10 dias de acompanhamento ${ }^{3,5}$.

Dois diagnósticos de enfermagem podem ser identificados nos cuidados de fim de vida de acordo com as alterações cognitivas e neurológicas: Confusão aguda e Confusão crônica, nesse estudo o diagnóstico encontrado foi o de Confusão crônica devido à prevalência de pacientes idosos com demência avançada. Entretanto, em pacientes oncológicos, a Confusão aguda, pode relacionar-se com o delirium comumente presente em fim de vida.

O controle e gerenciamento de sintomas no cuidado ao fim de vida na demência necessita da integração das avaliações das equipes especializadas em cuidados paliativos e cuidados gerontológicos e/ou cuidados a longo prazo $0^{3,16}$. Os cuidados integrados facilitam o gerenciamento de problemas comportamentais e a antecipação, avaliação e gerenciamento de problemas físicos e cognitivos, principalmente superando a dificuldade na comunicação dos sintomas esperados nos pacientes com demência avançada. Portanto os profissionais de saúde que atuam nessa área precisam de conhecimentos específicos de desconforto na alteração do comportamento ${ }^{3}$.

Portanto, a Síndrome de terminalidade na abordagem paliativista nos cuidados ao fim de vida na demência pode auxiliar no raciocínio, avaliação e gerenciamento de sintomas, que ocorrem simultaneamente. Durante a monitoração dos sintomas e abordagem ao paciente com demência avançada, o enfermeiro necessita ter a visão de apoio às famílias para ajudá-las em seu papel de tomadores de decisão e para auxiliá-los a lidar com uma alta carga de cuidado e luto antecipado causado pela contínua deterioração do paciente ${ }^{3,16}$.

Um estudo comparou os sintomas antes da morte em três grupos diferentes: pacientes com câncer, demência ou doença crônica, e as análises demostraram significativamente maiores em sintomas físicos para aqueles com demência e doença crônica no último mês de vida do que aqueles com câncer ${ }^{3}$. A dor é uma das causas de sofrimento, inquietação e distúrbios comportamentais em pacientes com demência, caracterizados por: comportamentos de agitação verbal como queixa, negativismo, frases e perguntas repetitivas, solicitação constante de atenção e xingamentos ou agressões verbais $^{17,18}$. A Dor crônica esteve presente em $44,7 \%$, o que se aproxima do estimado pela OMS de $47 \%$ para pacientes em cuidados ao fim de vida com demência ${ }^{2}$. Houve ainda alta frequência de Integridade tissular prejudicada e a Integridade da pele prejudicada (73,3\%) esperadas por associarem-se as condições nutricionais, idade avançada, incontinência, fragilidade capilar, baixa imunidade e mobilidade ${ }^{19-22}$. Além disso, as lesões em pacientes idosos com demência avançada são indicadores de cuidados ao fim de vida ${ }^{23}$.

Os diagnósticos psicosocioespirituais são inferiores nesse perfil populacional dado a maioria com perdas cognitivas severas, e, portanto, não permitem sua identificação. Como também pela oportunidade dos familiares ao longo do processo de doença elaborar as perdas, no entanto, em alguns casos podem ser prioritários, a depender do contexto familiar. Um estudo recente com o diagnóstico de Ansiedade relacionado à morte apresentou alta prevalência em cuidadores familiares de paciente paliativos oncológicos ${ }^{24}$. Porém, em análise de conjuntos diagnósticos, para compor a síndrome, identificou-se a necessidade de melhor diferenciação dos diagnósticos de Ansiedade e Pesar, do diagnóstico de Ansiedade relacionado à morte. 
A limitação encontrada para esse estudo foi o número amostral, no entanto trata-se de um serviço especializado, que justifica o extrapolamento dos dados. Os casos constituintes são inovadores, com poucos estudos sobre o tema no mundo e no Brasil. Entretanto se recomenda estudos em demais populações (oncológica, criança, cardiológica) e outros cenários para avaliar as variações do perfil diagnóstico em cada população.

\section{CONCLUSÃO}

Ao analisar a prevalência da Síndrome de terminalidade de forma conjunta nos últimos 10 dias de vida observouse a presença simultânea de em média 7,5 diagnósticos de enfermagem, portanto, recomenda-se a inclusão desse diagnóstico de enfermagem na Taxonomia II da NANDA-I.

\section{REFERÊNCIAS}

1. World Health Organization. National cancer control programmes: policies and managerial guidelines. Geneva (CH): WHO; 2002. [cited 2019 Jul 12]; 2eed: 15-16. Available from: https://apps.who.int/iris/bitstream/handle/10665/42527/9241590238.pdf

2. Worldwide palliative care alliance \& World Health Organization. Global atlas of palliative care at the end of life. London (UK): WPCA. 2014 [cited 2019 Jul 12]; 1-103. Available from: https://www.who.int/nmh/Global_Atlas_of_Palliative_Care.pdf

3. Boyd M, Frey R, Balmer D, Robinson J, McLeod H, Foster S, et al. End of life care for long-term care residents with dementia, chronic illness and cancer: prospective staff survey. BMC geriatrics [Internet]. 2019 [cited 2019 Jul 12]; 19(1):137. DOI: https://doi.org/10.1186/s12877-019-1159-2

4. Sleeman KE, Brito M, Etkind S, Nkhoma K, Guo P, Higginson IJ, et al. The escalating global burden of serious health-related suffering: projections to 2060 by world regions, age groups, and health conditions. The Lancet Global Health [Internet]. 2019 [cited 2019 Jul 12]; 7(7):e883-e892. English. DOI: https://doi.org/10.1016/S2214-109X(19)30172-X

5. Kydd A, Sharp B. Palliative care and dementia-A time and place?. Maturitas [Internet]. 2016 [cited 2019 Jul 12]; 84:5-10. DOI: https://doi.org/10.1016/j.maturitas.2015.10.007

6. Coleman AM. End-of-life issues in caring for patients with dementia: the case for palliative care in management of terminal dementia. American Journal of Hospice and Palliative Medicine ${ }^{\circledR}$ [Internet]. 2012 [cited 2019 Jul 12]; 29(1):9-12. DOI: https://doi.org/10.1177/1049909111410306

7. Lucena ADF, Barros ALBLD. Cross-mapping: an alternative to data analysis in nursing. Acta Paulista de Enfermagem [Internet] São Paulo. 2005 [cited 2019 Jul 12]; 18(1):82-8. DOI: http://dx.doi.org/10.1590/S0103-21002005000100011

8. Knaul FM, Farmer PE, Krakauer EL, Lima L, Bhadelia A, Kwete XJ, et al. Alleviating the access abyss in palliative care and pain relief-an imperative of universal health coverage: the Lancet Commission report. The Lancet [Internet]. 2017 [cited 2019 Jul 12]; 391(10128):1391-454. DOI: https://doi.org/10.1016/S0140-6736(17)32513-8

9. Ghosh A, Dzeng E, Cheng MJ. Interaction of palliative care and primary care. Clinics in geriatric medicine [Internet]. 2015 [cited 2019 Nov 20]; 31(2):207-18. DOI: http://dx.doi.org/10.1016/j.cger.2015.01.001

10. Akgün KM. Palliative and end-of-life care for patients with malignancy. Clinics in chest medicine [Internet]. 2017 [cited 2019 Nov 20]; 38(2):363-76. DOI: http://dx.doi.org/10.1016/j.ccm.2016.12.010

11. Li B, Mah K, Swami N, Pope A, Hannon B, Lo C, et al. Symptom Assessment in Patients with Advanced Cancer: Are the Most Severe Symptoms the Most Bothersome? Journal of palliative medicine [Internet]. 2019 [cited 2019 Nov 20]. 22(10). DOI: https://doi.org/10.1089/jpm.2018.0622

12. Swagerty D. Integrating Quality Palliative and End-of-Life Care into the Geriatric Assessment: Opportunities and Challenges. Clinics in geriatric medicine [Internet]. 2017 [cited 2019 Nov 20]; 33(3):415-29. DOI: https://doi.org/10.1016/j.cger.2017.03.005

13. Bamford C, Lee R, McLellan E, Poole M, Harrison-Dening K, Hughes J, et al. What enables good end of life care for people with dementia? A multi-method qualitative study with key stakeholders. BMC geriatrics [Internet]. 2018 [cited 2019 Nov 20]; 18(1):302. DOI: https://doi.org/10.1186/s12877-018-0983-0

14. Murphy E, Froggatt K, Connolly S, O'Shea E, Sampson EL, Casey D, et al. Palliative care interventions in advanced dementia. Cochrane database of systematic reviews [Internet]. 2016 [cited 2019 Nov 20]; 12:CD011513. DOI: https://doi.org/10.1002/14651858.CD011513.pub2

15. Fetherston AA, Rowley G, Allan CL. Challenges in end-of-life dementia care. Evidence-based mental health [Internet]. 2018 [cited 2019 Nov 20]; 21(3):107-11. DOI: http://dx.doi.org/10.1136/eb-2018-102889

16. Aldridge MD, Canavan M, Cherlin E, Bradley EH. Has hospice use changed? 2000-2010 utilization patterns. Medical care [Internet]. 2015 [cited 2019 Nov 20]; 53(1):95-101. DOI: https://doi.org/10.1097/mlr.0000000000000256

17. Husebo BS, Ballard C, Cohen-Mansfield J, Seifert R, Aarsland D. The response of agitated behavior to pain management in persons with dementia. The American Journal of Geriatric Psychiatry [Internet]. 2014 [cited 2019 Nov 22]; 22(7):708-17. DOI: http://dx.doi.org/10.1016/j.jagp.2012.12.006

18. Pandpazir M, Tajari M. The application of palliative care in dementia. Journal of family medicine and primary care [Internet]. 2019 [cited 2019 Nov 22]; 8(2):347-51. DOI: https://doi.org/10.4103/jfmpc.jfmpc_105_18

19. Ferris A, Price A, Harding K. Pressure ulcers in patients receiving palliative care: A systematic review. Palliative medicine [Internet]. 2019 [cited 2019 Nov 22]; 33(7):770-82. DOI: https://doi.org/10.1177/0269216319846023 
20. Kehl KA, Kowalkowski JA. A systematic review of the prevalence of signs of impending death and symptoms in the last 2 weeks of life. American Journal of Hospice and Palliative Medicine ${ }^{\circledR}$ [Internet]. 2013 [cited 2019 Nov 22]; 30(6):601-16. DOI: https://doi.org/10.1177/1049909112468222

21. Latimer S, Shaw J, Hunt T, Mackrell K, Gillespie BM. Kennedy Terminal Ulcers: A Scoping Review. Journal of Hospice \& Palliative Nursing [Internet]. 2019 [cited 2019 Nov 22]; 21(4):257-63. DOI: https://doi.org/10.1097/NJH.0000000000000563

22. Neloska L, Damevska K, Kuzmanova A, Pavleska L, Kostov M, Zovic BP. Dermatological diseases in palliative care patients: a prospective study of 271 patients. JDDG: Journal der Deutschen Dermatologischen Gesellschaft [Internet]. 2017 [cited 2019 Nov 22]; 15(6):621-7. DOI: https://doi.org/10.1111/ddg.13236

23. Rodríguez JR, Tabares VZ, Díaz CMG. Cuidados paliativos y envejecimiento. Geroinfo [Internet]. 2015 [cited 2019 Nov 22]; 10(3):1-21. Available from: https://www.medigraphic.com/pdfs/geroinfo/ger-2015/ger153b.pdf

24. Abreu-Figueiredo RM, Sá LO, Lourenço TM, Almeida SS. Death anxiety in palliative care: Validation of the nursing diagnosis. Acta Paul Enferm [Internet]. 2019 [cited 2019 Nov 22]; 32(2):178-85. DOI: https://doi.org/10.1590/1982-0194201900025 